\title{
Sky coverage and tip/tilt error analysis for TMT
}

\author{
Richard M. Clare ${ }^{a}$, Brent L. Ellerbroek ${ }^{a}$, Jean-Pierre Véran ${ }^{b}$, Glen Herriot $^{b}$, David Anderson ${ }^{b}$ \\ ${ }^{a}$ Thirty Meter Telescope Project, California Institute of Technology \\ 1200 E. California Boulevard, Mail Code 102-8, Pasadena, California 91125, USA \\ ${ }^{b}$ Herzberg Institute of Astrophysics, \\ 5071 West Saanich Road, Victoria, British Columbia, Canada
}

\begin{abstract}
A Monte Carlo sky coverage model for laser guide star adaptive optics systems is presented. This model provides fast Monte Carlo simulations of the tip/tilt (TT) wavefront error calculated with minimum variance estimators over natural guide star constellations generated from star models. With this simulation code we are able to generate a TT error budget for the Thirty Metre Telescope (TMT) facility Narrow Field Infra-Red Adaptive Optics System (NFIRAOS), and perform several design trade studies. With the current NFIRAOS design, the median TT error at the galactic pole with median seeing is calculated to be $65 \mathrm{~nm}$ or 1.8 mas.
\end{abstract}

Keywords: adaptive optics, wavefront sensing, sky coverage.

\section{INTRODUCTION}

Several extremely large telescopes (ELTs) of primary mirror diameters 20-100 m are currently in the planning and design stages, such as the Thirty Metre Telescope (TMT), ${ }^{1}$ the OverWhelmingly Large telescope (OWL), ${ }^{2}$ the Giant Magellan Telescope (GMT), ${ }^{3}$ and the Euro50 project. ${ }^{4}$ Successful utilization of the unprecedented resolution of these ELTs is contingent upon the correction of atmospheric turbulence with adaptive optics (AO). ${ }^{5}$

Several current AO systems ${ }^{6}$ employ a laser guide star (LGS) to provide wavefront sensor (WFS) measurements of the instantaneous wavefront aberrations without relying upon the availability of a bright natural guide star (NGS). However, because the laser jitters and is deflected on both the upward and downward paths through the atmosphere, the tip-tilt (TT) modes of the atmospheric aberration cannot be determined from the LGS. Consequently, NGS WFS(s) are also required to estimate these modes. The sky coverage problem is the probability of finding sufficiently bright NGS(s) within the isoplanatic patch of the science object that will allow accurate estimation of the TT modes.

An additional fundamental effect limiting LGS AO systems is tilt anisoplanatism, which arises when a single TT NGS is viewed off-axis with respect to the science object. For LGS multi-conjugate AO (MCAO), tilt anisoplanatism will degrade the uniformity of turbulence compensation over an extended field and may become the dominant wavefront error term. A number of different approaches to overcome tilt anisoplanatism have been proposed: (1) using multiple TT NGS, ${ }^{7}$ (2) using a NGS that measures TT and focus, ${ }^{8}$ and (3) using LGS at different altitudes ${ }^{7,9}$ (ie a combination of Rayleigh and sodium LGS). Because the altitude of the sodium layer is constantly changing, ${ }^{10}$ and it is not possible to disentangle atmospheric focus aberrations from these altitude variations with the LGS measurements, we modify option (1) to be multiple TT sensors and a TT-focus sensor. In this paper, we will evaluate the performance of these three options.

In Ref. 11, a method for producing Monte Carlo sky coverage simulations over random NGS constellations is presented. The essence of this method is to propagate the phase screens at each altitude, which are represented as a Zernike basis sum, to the aperture using geometric optics and taking into account the cone effect for the finite height of the LGSs, as well as the tilt anisoplanatism caused by the GSs being off-axis with respect to the science object. The expected wavefront error is then calculated using a minimum variance estimator from these transformation matrices and the statistical properties of the atmosphere.

Further author information: Send correspondence to R.M.C.

Ph: 626-295-1621,

Email: rclare@caltech.edu

Advances in Adaptive Optics II, edited by Brent L. Ellerbroek, Domenico Bonaccini Calia, Proc. of SPIE Vol. 6272, 627232, (2006) · 0277-786X/06/\$15 - doi: 10.1117/12.669680 
In Ref. 12, a number of practical considerations are incorpoareted into the sky coverage model. These include (i) guide star statistics using the Bahcall-Soneira and Spagna models, (ii) noise on the NGS measurements, (iii) a telescope wind-shake model, (iv) a model for how the Strehl and hence NGS WFS measurement noise varies across the field in the infra-red (IR), (v) the error due to imperfectly tracking the range to the sodium layer, (vi) the mechanical bandwidths of the TT stage and deformable mirror (DM) actuators, and (vii) temporal filtering of the NGS measurements to balance the errors due to wind-shake, noise, servo lag and sodium altitude tracking.

In this paper, we summarize the work in Ref.'s 11 and 12 and employ the sky coverage model to perform a number of design trades for Narrow Field Infra-Red Adaptive Optics System (NFIRAOS). ${ }^{13}$ These include whether to use optical (V band) or IR ( $\mathrm{J}$ band) stars and sensors for the NGS WFS. Secondly, we find the optimal patrol field diameter required to find sufficient NGS. Thirdly, we use this methodology to evaluate the different methods for correcting tilt anisoplanatism described previously. We also compare the performance of a quad-cell Shack-Hartmann (SH) detector with a matched-filter or noise-weighted least squares approach for measuring TT from the NGS. The theory of the sky coverage simulator, including the transformation matrices, control alogirthm and minimum variance reconstructors, is presented in Section 2. Some of the computational details of the sky coverage simulator are outlined in Section 3. Monte-Carlo simulation results for NFIRAOS are presented in Section 4. Conclusions are drawn in Section 5.

\section{THEORY}

\subsection{Background and notation}

We consider the atmosphere to consist of $N_{l}$ discrete layers of turbulence. We model the phase screen at the $i^{t h}$ atmospheric layer, $\phi(i ; r, \theta)$, as a finite sum of $N$ orders of Zernike polynomials, ${ }^{14}$

$$
\phi(i ; r, \theta)=\sum_{n=0}^{N} \sum_{m=0}^{n} a_{n m}(i) Z_{n m}(r, \theta),
$$

where $Z_{n m}$ is the $m^{t h}$ Zernike polynomial of radial order $n$, and $a_{n m}(i)$ are the coefficients of the corresponding Zernike polynomials at the $i^{\text {th }}$ layer.

For each WFS in the AO system, the phase screens at each atmospheric layer, $a$, are projected to the aperture plane by a transformation matrix, $T$, such that

$$
b=T a,
$$

where $b$ are the Zernike coefficients of the wavefront measured by the WFS, and

$$
a=\left(\begin{array}{c}
a(1) \\
\vdots \\
a(i) \\
\vdots \\
a\left(N_{l}\right)
\end{array}\right) .
$$

The $T$ matrix takes into account the off-axis effect of the NGS or LGS, as well as the cone effect due to the finite height of the LGS, and also includes summation over all of the atmospheric layers. The exact form of the $T$ matrix is defined in Ref. 11. The wavefront modes as seen by the LGS, $b_{L}$, (both sodium, and when applicable, Rayleigh) are given by

$$
b_{L}=T_{L} a,
$$

where the $L$ subscript in this paper refers to the LGS. In this paper, the LGS measurements are assumed to be noiseless and instantaneous, since ultimately we are primarily concerned with the performance of the system with respect to the NGS, and only the lower-order component of the TT-removed LGS measurements are considered (typically Zernike radial orders 2 through 6 ).

Similarly, the wavefront modes seen from the NGS, $b_{n}$, are given by

$$
b_{n}=T_{n} a^{\prime}+n^{\prime} .
$$


where the $n$ subscript refers to the NGS, and the vector $n^{\prime}$ is additive noise on the NGS WFS measurements. The prime $(')$ notation on $a$ and $n$ is to show that the NGS measurements, $b_{n}$, are temporally filtered by a controller $H_{o l}(s)$ to minimize the combined effect of the measurement noise and the servo lag.

The Zernike coefficients, $b_{s}$, of the wavefronts projected into the aperture plane for sources in the directions of the science evaluation points, are given by

$$
b_{s}=T_{s} a,
$$

where the $s$ subscript refers to the science field. The $N_{s}$ science evaluation points we consider for NFIRAOS are defined in Section 4.

Finally, the deformable mirrors (DMs) provide a correction to the wavefronts associated with the science evaluation directions that is given by

$$
b_{m}=T_{m} P_{m} \hat{a},
$$

where the $m$ subscript refers to mirrors throughout this paper, and $P_{m}$, the optimal fit of Zernike modes to the DM's to compensate the estimated science phase profiles, is defined in Ref. 11 by

$$
P_{m}=\left(T_{m}{ }^{T} W T_{m}\right)^{-1} T_{m}{ }^{T} W T_{s} .
$$

Here $W$ is a block diagonal weighting matrix of $N_{s}$ blocks whose elements are $w_{k} I$, where $I$ is an identity matrix of dimension equal to the number of Zernike modes computed for each science wavefront. If the entire science field is considered, $w_{k}=1 / N_{s}$ for all $k$ points. If only the on-axis science point is considered, $w_{1}=1$ and $w_{k}=0$ otherwise.

Similar mirror transformation matrices can be derived for the correction of the NGS modes, $T_{m, n}$, and the correction of the LGS modes, $T_{m, L}$.

\subsection{Control algorithm overview}

The control algorithm we employ in this paper is a two step process. We first estimate the wavefront Zernike coefficients at each layer, $a$, and the NGS measurements, $b_{n}$, from the LGS measurements, $b_{L}$. We then estimate the residual uncertainty in $a, a_{\text {res }}$, from the new information provided by the actual NGS measurements, $b_{n}$. It may be shown that this two step approach is equivalent to an integrated one step minimum variance estimator, but the two step approach is a more efficient approach to Monte-Carlo simulations of NGS constellations. The control model is shown in Fig. 1, and is described in more detail below.

Firstly, the Zernike coefficients at each of the atmospheric layers are estimated from the LGS measurements, $b_{L}$, using

$$
\begin{aligned}
\hat{a}_{L} & =E_{L} b_{L} \\
& =E_{L} T_{L} a
\end{aligned}
$$

where $E_{L}$ is the minimum variance estimator of the atmospheric modes, $a$, from the LGS measurements, $b_{L}$. The residual error in this estimate is given by

$$
a_{\mathrm{res}}=a-\hat{a}_{L},
$$

and from Fig. 1, the estimate of the residual error (computed from the NGS measurements) is related to the final estimate $\hat{a}$ by

$$
\hat{a}_{\text {res }}=\hat{a}-\hat{a}_{L} .
$$

Note that although Eq. (9) indicates that the LGS WFS measurement vector $b_{L}$ is measured in open loop without the corrections applied by the DMs, NFIRAOS will actually generate "pseudo-open-loop" measurements by combining closed-loop measurements with knowledge of DM actuator commands, as illustrated in Fig. 1. Secondly, the residual uncertainty, $a_{\text {res }}$, is estimated based upon the new information provided by the temporally filtered NGS WFS measurement.

Temporal filtering is applied to balance the estimation errors due to NGS noise, servo lag, sodium layer altitude uncertainty and wind-shake. The NFIRAOS TT control system utilizes both a TT stage (TTS) and 


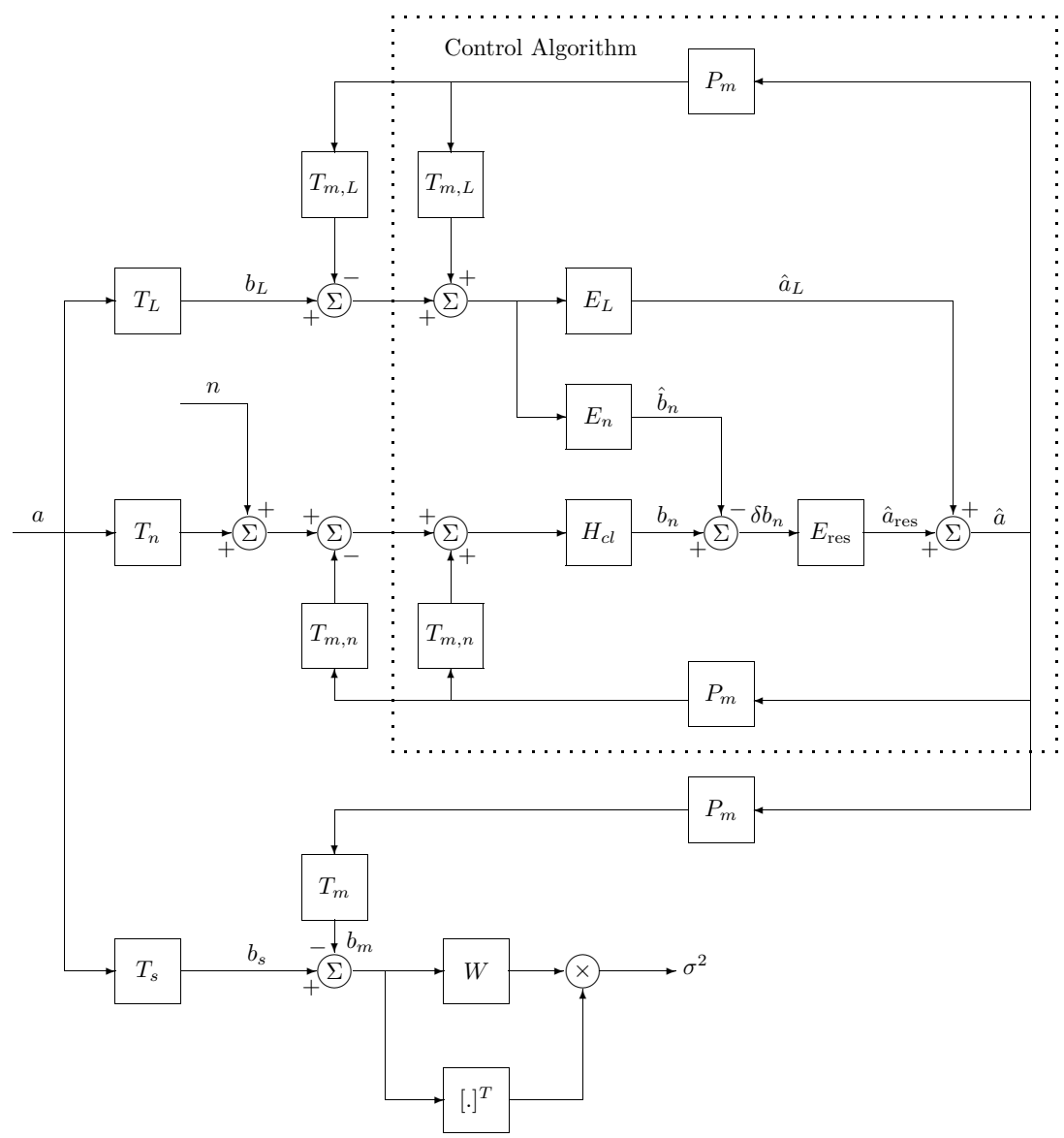

Figure 1. The adaptive optics control system model used in this paper. See the text for the matrix definitions.

the DM actuators in a "woofer-tweeter" architecture, due to the bandwidth and stroke limitations, respectively, of these two sets of actuators. The open-loop temporal filter, $H_{o l}(s)$, where $s$ is the Laplace coordinate, is the sum of three terms: the DM transfer function, and the proportional and integral control terms of the TTS. The filtered NGS WFS measurement is given by

$$
\begin{aligned}
b_{n} & =\frac{H_{o l}(s)}{1+H_{o l}(s)}\left[T_{n} a+n\right] \\
& =H_{c l}(s)\left[T_{n} a+n\right] \\
& =T_{n}\left[H_{c l}(s) a\right]+H_{c l}(s) n \\
& =T_{n} a^{\prime}+n^{\prime},
\end{aligned}
$$

where $H_{c l}(s)$ is the closed-loop temproal filter. The "new information" provided by this measurement is the component not predicted by the LGS WFS measurement,

$$
\begin{aligned}
\delta b_{n} & =b_{n}-\hat{b}_{n} \\
& =b_{n}-E_{n} b_{L},
\end{aligned}
$$

where $E_{n}$ is the minimum variance estimator for the NGS measurements $b_{n}$ from the LGS measurements, $b_{L}$. Combining Eq.'s (16) and (18) yields

$$
\delta b_{n}=T_{n} a^{\prime}+n^{\prime}-E_{n} T_{L} a .
$$


Finally, the estimate of the residual uncertainty $\hat{a}_{\text {res }}$ is given by the expression

$$
\hat{a}_{\text {res }}=E_{\text {res }} \delta b_{n},
$$

where $E_{\text {res }}$ is the minimum variance estimator of the estimate of the residual atmospheric modes from the new information in the NGS measurements, $\delta b_{n}$. The minimum variance estimators $E_{L}, E_{n}$, and $E_{\text {res }}$, are derived in Ref. 12.

\subsection{Minimum variance estimators}

The covariance matrix of the atmospheric modes, $C_{\phi}=\left\langle a a^{T}\right\rangle$, is a block diagonal matrix, with the $\mathrm{i}^{\text {th }}$ block representing the covariance of the $\mathrm{i}^{\text {th }}$ layer of the atmosphere,

$$
C_{\phi}=\left(\begin{array}{ccccc}
C_{\phi}(1) & & & & 0 \\
& \ddots & & & \\
& & C_{\phi}(i) & & \\
& & & \ddots & \\
0 & & & & C_{\phi}\left(N_{l}\right)
\end{array}\right),
$$

where there are $i=1 \ldots N_{l}$ layers of turbulence, and the covariance of the $i^{t h}$ layer is given by

$$
C_{\phi}(i)=\left\langle a(i) a(i)^{T}\right\rangle=\gamma(i) C,
$$

$\gamma(i)$ is the strength of layer $i$, and $C$ is a normalized covariance matrix for a single phase screen with unit strength. Similarly, the covariance of the filtered atmospheric modes with the non-filtered atmospheric modes, $C_{\phi}^{\prime}=\left\langle a^{\prime} a^{T}\right\rangle$, and the covariance of the filtered atmospheric modes, $C_{\phi}^{\prime \prime}=\left\langle a^{\prime} a^{\prime T}\right\rangle$, are block diagonal matrices. See Ref. 12 for the derivation of $C_{\phi}^{\prime}$ and $C_{\phi}^{\prime \prime}$.

The covariance of the temporally filtered noise, $C_{N}$, is given by

$$
C_{N}=\left\langle n^{\prime} n^{\prime T}\right\rangle .
$$

$C_{N}$ is derived for a quad-cell Shack-Hartmann WFS and for a matched-filter (noise-weighted least squares) algorithm in Ref. 12.

The mean-squared difference between the atmospheric modes, $a$, and the estimated atmospheric modes, $\hat{a}_{L}$, and the NGS measurements, $b_{n}$, and the estimate of the NGS measurements, $\hat{b}_{n}$, is ${ }^{12}$

$$
\begin{aligned}
\left\langle\left(\begin{array}{c}
a-\hat{a}_{L} \\
b_{n}-\hat{b}_{n}
\end{array}\right)\left(\begin{array}{c}
a-\hat{a}_{L} \\
b_{n}-\hat{b}_{n}
\end{array}\right)^{T}\right\rangle & =\left(\begin{array}{cc}
C_{\phi}-E_{L} T_{L} C_{\phi} & C_{\phi}^{\prime} T_{n}{ }^{T}-C_{\phi} T_{L}^{T} E_{n}{ }^{T} \\
T_{n} C_{\phi}^{\prime}-E_{n} T_{L} C_{\phi} & T_{n} C_{\phi}^{\prime \prime} T_{n}^{T}-E_{n} T_{L} C_{\phi}^{\prime} T_{n}^{T}+C_{N}
\end{array}\right) \\
& \equiv\left(\begin{array}{cc}
A & B^{T} \\
B & D+C_{N}
\end{array}\right) .
\end{aligned}
$$

The mean-squared difference between the residual atmospheric modes, $a_{\text {res }}$, and the estimate of the residual atmospheric modes, $\hat{a}_{\text {res }}$, is given by

$$
\begin{aligned}
\left\langle\left(a_{\text {res }}-\hat{a}_{\text {res }}\right)\left(a_{\text {res }}-\hat{a}_{\text {res }}\right)^{T}\right\rangle & =A-E_{\text {res }} B-B^{T} E_{\text {res }}^{T}+E_{\text {res }}\left(D+C_{N}\right) E_{\text {res }}^{T} \\
& =A-B^{T}\left(D+C_{N}\right)^{-1} B .
\end{aligned}
$$

Subtracting Eq. (12) from Eq.(11) shows that

$$
a-\hat{a}=a_{\mathrm{res}}-\hat{a}_{\mathrm{res}},
$$

so that Eq. (25) actually describes the overall estimation error in the two step estimation algorithm,

$$
\left\langle(a-\hat{a})(a-\hat{a})^{T}\right\rangle=A-B^{T}\left(D+C_{N}\right)^{-1} B .
$$




\subsection{Residual phase variance formulae}

The estimation error covariance matrix $\left\langle(a-\hat{a})(a-\hat{a})^{T}\right\rangle$ defines the error in estimating the atmospheric turbulence profile, not the wavefront error for the science instrument. In Ref. 11, we derive the mean-squared wavefront error $\left(\operatorname{rad}^{2}\right)$ for a single conjugate $\mathrm{AO}$ system as

$$
\sigma^{2}=\operatorname{Tr}\left[T_{s}^{T} W T_{s}\left\langle(a-\hat{a})(a-\hat{a})^{T}\right\rangle\right],
$$

where $\operatorname{Tr}$ is the trace of the matrix. If we substitute Eq. (28) for $\left\langle(a-\hat{a})(a-\hat{a})^{T}\right\rangle$ in Eq. (29), we obtain

$$
\sigma^{2}=\operatorname{Tr}\left[T_{s}^{T} W T_{s}\left(A-B^{T}\left(D+C_{N}\right)^{-1} B\right)\right] .
$$

The wavefront error, $\sigma$, in nanometers of phase is often more convenient. The conversion from $\sigma^{2}\left(\operatorname{rad}^{2}\right)$ to $\sigma(\mathrm{m})$ is simply

$$
\sigma(m)=\frac{\sqrt{\sigma^{2}\left(\operatorname{rad}^{2}\right)}}{2 \pi / \lambda_{e}},
$$

where $\lambda_{e}$ is the $r_{0}$ evaluation wavelength.

In Ref. 12, formulae to deconstruct the TT error into terms for servo lag, NGS WFS noise and tilt anisoplanatism are derived.

\section{COMPUTATIONAL DETAILS}

In this section, we discuss the important computational details required to implement the theory persented in the previous section. See Ref. 12 for the calculation of the temporally filtered atmospheric covariance matrices and the noise covariance matrices for both the quad-cell Shack-Hartmann and the matched filter approaches.

\subsection{Star generation}

In V band, the NGS are generated using the Bahcall-Soneira star model. ${ }^{15}$ This produces stars up to a magnitude of 30. In J band, the NGS are generated using the Spagna model, ${ }^{16}$ which produces stars with magnitude up to 22 at the North Galactic Pole (NGP), and 19 for other galactic latitudes.

\subsection{NGS sharpening}

The NGS are expected to be partially corrected (sharpened) by the AO system in J band. The Strehl of each NGS is a function of its position in the field. Strehl ratios were generated in the adaptive optics simulator $\mathrm{LAOS}^{17}$ at five points along the $\mathrm{x}$ axis, and 4 points along a line at 30 degrees to the $\mathrm{x}$ axis, although as shown in Fig. 2(a), there is little angular dependence on the Strehl, and the angular dependence is ignored. A cubic fit was made to these 9 points, as shown in Fig. 2(a). These J band Strehls are for the baseline version of NFIRAOS, and better partial correction is expected for the upgrade version of NFIRAOS, which would provide a higher-order of wavefront correction.

\subsection{Wind-shake}

In this paper, the wind-shake is modeled as being statistically independent from atmospheric turbulence, so the total TT error is the sum of the contributions of the two effects. Initial simulations showed that the wind-shake term was the dominant term in the TT error budget for NFIRAOS and that the tip-tilt stage (TTS) alone was insufficient to correct for the TT induced from the wind-shake. Instead, we use both the TTS and the DM to correct for the wind-shake (woofer-tweeter control). Additionally, we control the TTS with a PI (proportional integral) controller, such that there are three control paths: the DM, the TTS with a single integrator, and the TTS with two integrators. Each path has a separate gain which is dependent on the sampling frequency. The woofer-tweeter control of the DM and TTS for NFIRAOS is described in Ref. 18.

The level of uncorrected wind-shake assumed is 25 mas, which is the median expected value for the telescope in median wind conditions. ${ }^{19}$ The TTS is assumed to have a $20 \mathrm{~Hz}$ mechanical bandwidth. The residual telescope wind-shake TT jitter after correction by the TTS and DM, $\sigma_{\text {res }}^{2}$, for this model is shown in Fig. 3(a). The discontinuous nature of this curve is due to the constraints chosen to optimize the gains of the controller. 


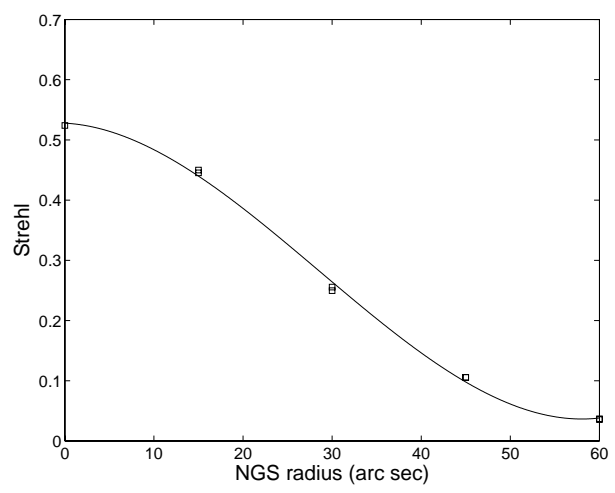

(a)

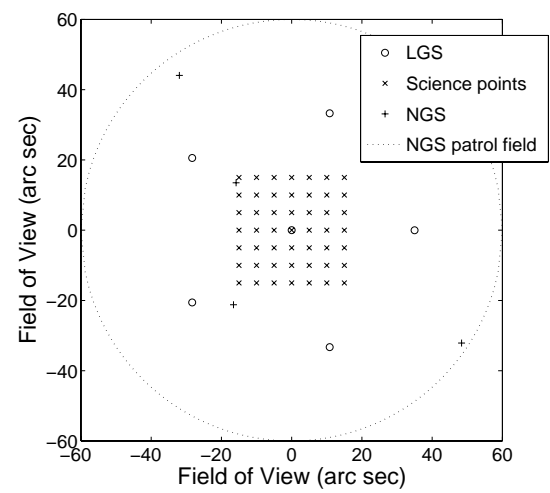

(b)

Figure 2. (a)The Strehl ratio as a function of the position of the NGS in the field. The 'o' points represent the Strehls generated using LAOS and the line is a best fit cubic approximation. (b) The field of view for NFIRAOS showing the sodium LGS (o), the 2 arc min diameter patrol field for the NGS (dotted line), and a random constellation of 4 NGS (+), and the science evaluation points $(\mathrm{x})$ for the 30 arc sec square science field.

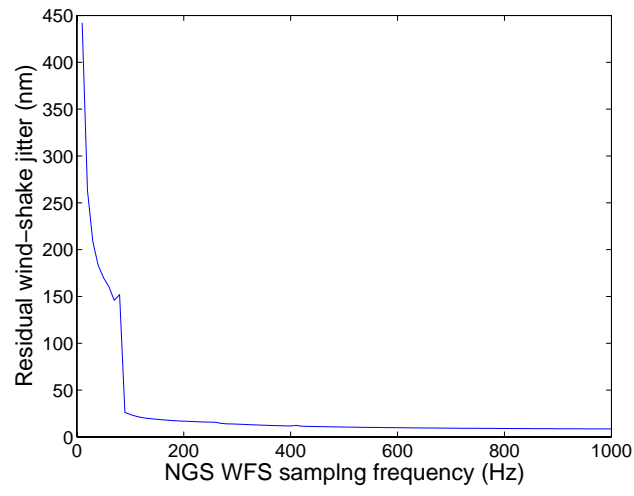

(a)

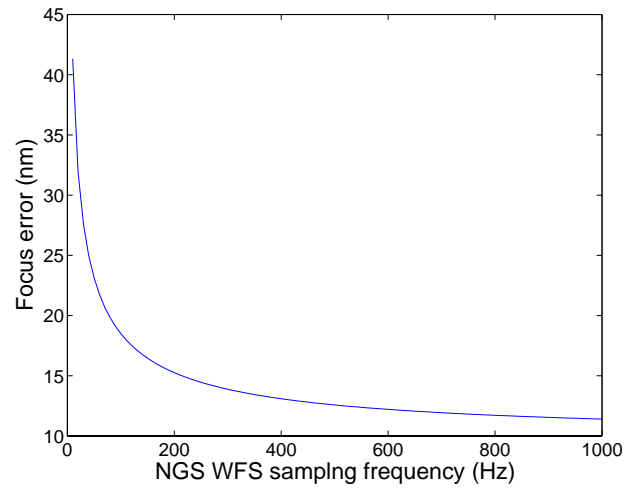

(b)

Figure 3. (a)The residual telescope wind-shake tilt jitter after correction as a function of the sampling frequency of the NGS WFS. (b) The estimated focus wavefront error due to variations in the mean altitude of the sodium layer as a function of the sampling frequency of the NGS WFS.

\subsection{Sodium layer range estimation error}

Experimental results have shown that the mean altitude of the sodium layer can vary by several metres per second. ${ }^{10}$ This temporal variation of the sodium layer results in a focus error, which cannot be determined from the sodium LGS WFS because it cannot be disentangled from the atmospheric focus aberration. Therefore one of the NGS is required to measure the focus term, and the sampling rate of the TTFA NGS WFS determines the focus error from the sodium layer altitude variations. So although the purpose of this paper is to estimate TT errors and not higher order errors such as this focus error, we also include this error when we optimize the sampling frequency of the NGS WFS. 
Table 1. Six layer turbulence profile, typical of Cerro Pachon, ${ }^{21}$ showing the height $h(i)$ of each layer, the relative turbulence strength, $\gamma(i)$, and windspeed, $v(i)$.

\begin{tabular}{cccc}
\hline Layer $(i)$ & $h(i)(\mathrm{m})$ & $\gamma(i)$ & $v(i)\left(\mathrm{ms}^{-1}\right)$ \\
\hline 1 & 0 & 0.6523 & 5 \\
2 & 2577 & 0.1723 & 13 \\
3 & 5155 & 0.0551 & 20 \\
4 & 7732 & 0.0248 & 30 \\
5 & 12887 & 0.0736 & 20 \\
6 & 15464 & 0.0219 & 10 \\
\hline
\end{tabular}

The calculation of the error in tracking the mean altitude of the sodium layer is presented in Ref. 20. This focus error calculation is made by extrapolating a power spectrum of height variations from Lidar data, and applying the rejection transfer function for using electronic offsets, which will correct for the focus of the LGS WFSs in real-time. The residual wavefront focus error, $\sigma_{N a}$, is plotted versus NGS WFS sampling frequency in Fig. 3(b) for the median observed sodium altitude variations.

\subsection{Sampling frequency optimization}

The overall wavefront error, $\sigma^{2}\left(\operatorname{rad}^{2}\right)$, is the sum of the atmospheric error, $\sigma_{a t m}^{2}$, which is computed with Eq. (30), the residual telescope windshake jitter, $\sigma_{r e s}^{2}$, and the sodium layer tracking error, $\sigma_{N a}^{2}$,

$$
\sigma^{2}\left(f_{s}\right)=\sigma_{a t m}^{2}\left(f_{s}\right)+\sigma_{r e s}^{2}\left(f_{s}\right)+\sigma_{N a}^{2}\left(f_{s}\right) .
$$

All the error terms in Eq. (32) are functions of the sampling rate, $f_{s}$. We optimize the sampling frequency for each NGS constellation with

$$
f_{s}^{*}=\arg \min _{f_{s}}\left\{\sigma_{\text {atm }}^{2}\left(f_{s}\right)+\sigma_{r e s}^{2}\left(f_{s}\right)+\sigma_{N a}^{2}\left(f_{s}\right)\right\}
$$

\section{NFIRAOS SIMULATIONS}

In this section, the sky coverage simulations for the AO system NFIRAOS at the NGP $\left(\right.$ latitude $=0^{\circ}$, longitude $=90^{\circ}$ ), which represents the worst case for sky coverage, are presented. The turbulence and wind velocity profile used in these simulations is tabulated in Table 1. This profile is generated from measurements obtained at Cerro Pachon. ${ }^{21}$ The other atmospheric and telescope parameters are tabulated in Table 2.

The wavefront errors are evaluated on-axis for a single conjugate AO system using Eq. (32). NFIRAOS in fact has a 10 arc sec square field in the baseline design, and 30 arc square field in the upgrade path, although overall TT performance for these fields is typically within 4 percent of the on-axis case.

We investigate both optical (V band) and IR ( $\mathrm{J}$ band) sensing for NFIRAOS. We assume the pixels in J band are twice diffraction-limited (ie $w$ in $\mathrm{J}$ band is $\lambda / D$ rads $=0.0086$ arc sec) and seeing-limited in $\mathrm{V}$ band $(w=0.5 \mathrm{arcsec})$. The levels of the read noise, $\sigma_{e}$, considered are $0,5,10$ and 15 electrons per pixel per readout. Although the first 6 Zernike orders are considered in the problem, only the errors arising in the TT terms are evaluated.

The sampling rate of the NGS WFS, $f_{s}$, is optimized for each NGS configuration. The allowable range of sampling frequencies is 10 to $1000 \mathrm{~Hz}$. A single simulation of one NGS configuration takes of the order of two seconds. The majority of this time is spent in optimizing the sampling frequency. The wavefront error, $\sigma^{2}$, for each element in the simulation space is computed for 500 NGS constellations. By re-seeding the random number generator, every option in the simulation space is simulated over the same set of 500 NGS constellations.

The baseline NFIRAOS LGS asterism is shown in Fig. 2(b), and consists of six LGS: one LGS is on-axis and the remaining five are equally spaced on a ring of diameter 70 arc sec. The Rayleigh LGS, when used, is on-axis, and at a range of $20 \mathrm{~km}$. The science field consists of 49 points arranged in a square grid of linear dimension 10 arc sec in the baseline design and 30 arc sec in the upgrade path. 
Table 2. System and atmospheric parameters.

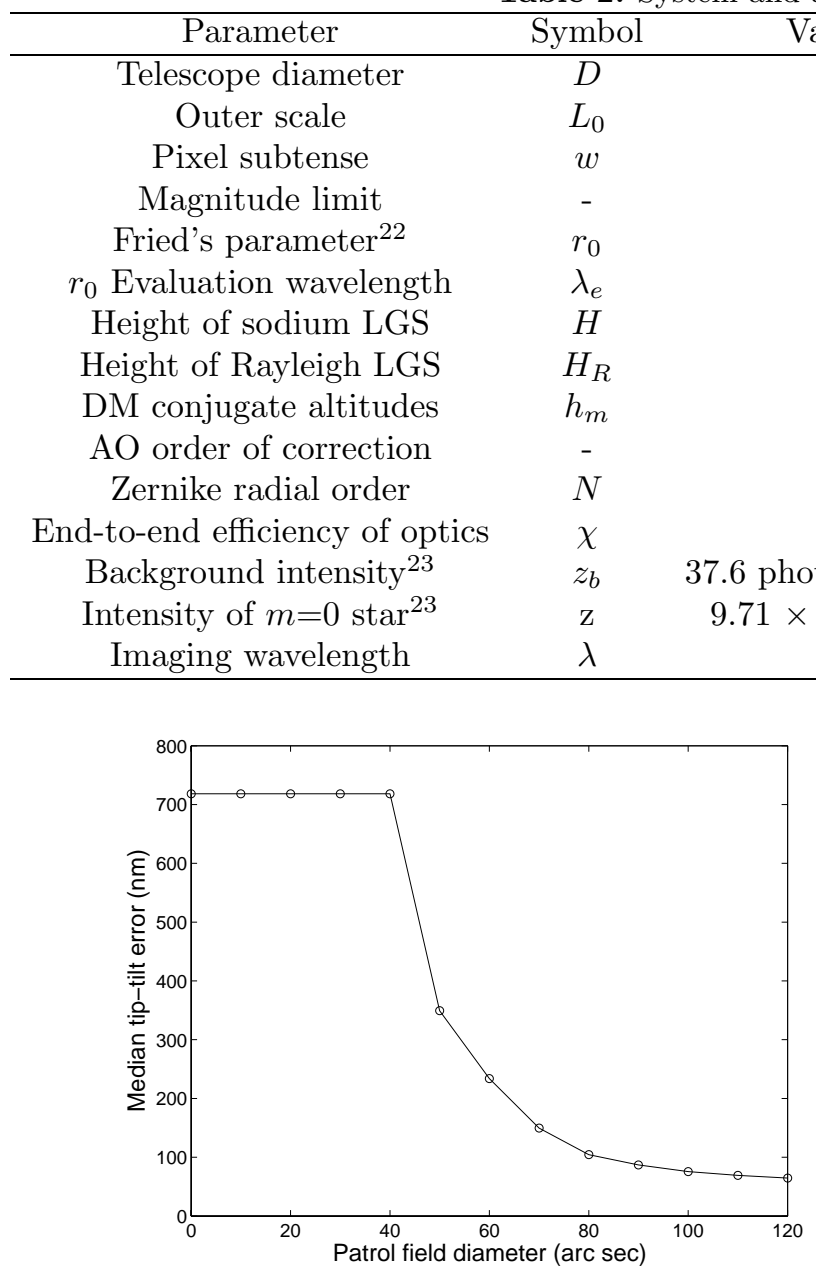

(a)

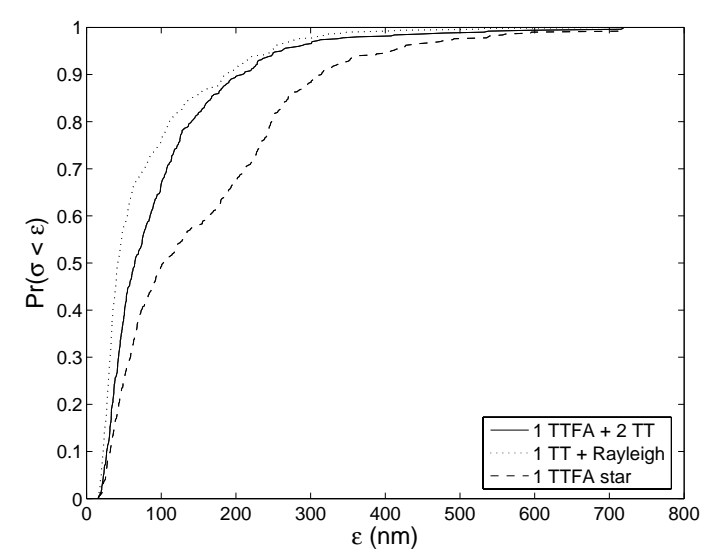

(b)

Figure 4. (a)The median TT error (nm) over 500 NGS constellations versus the patrol field diameter (arc sec) for 2 TT NGS and 1 TTFA NGS in J band. (b) The CDF $(\operatorname{Pr}(\sigma<\epsilon)$ vs $\epsilon(\mathrm{nm}))$ from 500 different NGS constellations in J band with 10 electrons of read noise for NFIRAOS for 1 TTFA NGS WFS and 2 TT NGS WFS (solid line), a single TTFA NGS WFS (dashed), and a single TT NGS WFS used in conjunction with a Rayleigh LGS (dotted).

The Bahcall-Soneira and Spagna models can generate more NGS than there are NGS WFS in the NFIRAOS design options. If there are more NGS in the field than NGS WFS, the wavefront error for every combination of NGS is evaluated, and the NGS combination that produces the smallest wavefront error is chosen.

The median TT error versus patrol band diameter is shown in Fig. 4(a). When the patrol diameter is less than or equal to $40 \mathrm{arc} \mathrm{sec}$, the median case corresponds to zero NGS in the field, and a TT error of $718 \mathrm{~nm}$. The lowest wavefront error is effectively obtained with a 2 arc min diameter field patrol field; stars further away from the science field effectively suffer too much anisoplanatism and the Strehl is too small to be useful. We use this diameter for the remainder of the simulations.

In this section, the TT errors are reported in $\mathrm{nm}$ rms. It is possible to convert the reported errors from $\mathrm{nm}$ 
Table 3. Median TT errors (nm) for NFIRAOS for the three NGS WFS options with Shack-Hartmann sensors using quad-cell detectors.

\begin{tabular}{cccccc}
\hline \multirow{2}{*}{ Band } & \multirow{2}{*}{ WFS option } & \multicolumn{4}{c}{ TT error $(\mathrm{nm})$} \\
\cline { 3 - 6 } & 1 TTFA + 2 TT & 371 & 374 & 377 & 386 \\
& 1 TTFA & 395 & 398 & 409 & 426 \\
V band & 1 TT + Rayleigh & 387 & 388 & 391 & 396 \\
& & & & & \\
& 1 TTFA + 2 TT & 43 & 54 & 65 & 76 \\
J band & 1 TTFA & 67 & 82 & 103 & 128 \\
& 1 TT + Rayleigh & 37 & 39 & 42 & 45 \\
\hline
\end{tabular}

Table 4. "Median" TT errors (nm) for NFIRAOS broken down into wind-shake, noise, tilt anisoplanatism and servo lag for the three WFS options in J band. There are 10 electrons of read noise per pixel.

\begin{tabular}{cccc}
\hline \multirow{2}{*}{ Error source } & \multicolumn{3}{c}{ Median TT error $(\mathrm{nm})$} \\
\cline { 2 - 4 } & 1 TTFA +2 TT & 1 TTFA & 1 TT + 1 Rayleigh LGS \\
\hline Wind-shake & 26 & 26 & 21 \\
Servo lag & 15 & 15 & 14 \\
Tilt anisoplanatism & 37 & 54 & 21 \\
NGS WFS noise & 44 & 84 & 26 \\
Total & 65 & 104 & 42 \\
\hline
\end{tabular}

to tilt jitter in mas by

$$
\sigma(\text { mas })=\frac{4 \times 1000 \times 180 \times 60 \times 60}{D \pi} \sigma(m)
$$

For a 30m diameter telescope, 1 mas of TT jitter corresponds to $36.4 \mathrm{~nm}$ TT error.

The median TT errors for the three WFS options in J and V bands are displayed in Table 3 for $0,5,10$ and 15 electrons of read noise per pixel. The first thing we note from Table 3 is that the results using seeing-limited stars in $\mathrm{V}$ band are, in all cases, significantly worse than using partially compensated stars in J band in terms of $\sigma(\mathrm{nm})$. We therefore eliminate using $\mathrm{V}$ band stars and sensors, and concentrate on $\mathrm{J}$ band for the remainder of this paper.

There is a clear hierarchy in the performance of the three WFS architectures, with the auxiliary Rayleigh LGS the best at all read noise levels, followed by the TTFA and two TT NGS WFS option, and lastly the single TTFA NGS WFS. This introduces a cost versus performance trade-off in the system design, with the Rayleigh LGS obviously being significantly more complex than the other two options.

The cumulative density function $(\mathrm{CDF}), \operatorname{Pr}(\sigma<\epsilon)$ vs $\epsilon(\mathrm{nm})$, is shown for the three WFS options in $J$ band in Fig 4, with 10 electrons of read noise, which is the baseline value. We see from Fig. 4 that the auxiliary Rayleigh LGS option always provides a lower TT error than the 1 TTFA + 2 TT NGS WFS option, which always produces a lower TT error than 1 TTFA NGS WFS.

In Table 4, the "median" TT error is broken down into wind-shake, servo lag, tilt anisoplanatism and noise on the NGS WFS measurements for the three WFS options with 10 electrons of read noise per pixel. Here the "median" TT error is the error term averaged over the 20 middle NGS constellations sorted on total TT error. The median sampling rate is $90 \mathrm{~Hz}$ for the 2 TT NGS WFS + 1 TTFA NGS WFS option, $90 \mathrm{~Hz}$ for the 1 TTFA NGS WFS, and $140 \mathrm{~Hz}$ for the auxiliary Rayleigh option. The NGS WFS noise is currently the largest term in the error budget for all options, followed by tilt anisoplanatism, wind-shake and servo lag. For the 2 TT NGS WFS + 1 TTFA NGS WFS and 1 TTFA NGS WFS options, the "median" sodium tracking error is 19 nm, and $17 \mathrm{~nm}$ for the auxiliary Rayleigh LGS option.

Finally, we compare the performance of the SH WFS quad-cell with a matched filter for three TT NGS WFS. We also investigate different pixel widths in conjunction with the matched filter approach: $\lambda / 2 D, \lambda / D$, and $3 \lambda / 2 D$. The median total TT errors for the matched filter, and also for the quad-cell as a comparison, are 
Table 5. Median TT errors (nm) for NFIRAOS with three TT NGS WFS for the quad-cell and matched filter approaches.

\begin{tabular}{cccccc}
\hline \multirow{2}{*}{ Detection method } & $\begin{array}{c}\text { Pixel subtense } \\
\text { (rads) }\end{array}$ & \multicolumn{4}{c}{ Median TT error (nm) } \\
\cline { 3 - 6 } & $\lambda / D$ & $\sigma_{e}=0$ & $\sigma_{e}=5$ & $\sigma_{e}=10$ & $\sigma_{e}=15$ \\
\hline Quad-Cell & $\lambda / 2 D$ & 39 & 65 & 80 & 93 \\
Matched filter & $\lambda / D$ & 32 & 53 & 70 & 79 \\
Matched filter & $3 \lambda / 2 D$ & 33 & 46 & 56 & 66 \\
Matched filter & & & & 57 & 68 \\
\hline
\end{tabular}

displayed in Table 5. The matched filter approach produces lower TT errors than the quad-cell for all pixel sizes and read noise levels investigated. The optimal pixel size is $\lambda / 2 D$ for 0 electrons of read-noise per pixel, and $\lambda / D$ for 5,10 and 15 electrons of read noise. The improvement with the matched filter is not due to the noise term alone. Although the matched filter approach produces less noise, this can allow a faster sample rate to reduce wind-shake and servo lag, and can allow an NGS constellation with less tilt anisoplanatism to be chosen. Using the matched filter algorithm is contingent upon improvements in IR technology to allow large, low noise, IR detector arrays.

\section{CONCLUSIONS}

In this paper, we have presented the modelling of sky coverage for an ELT, in particular the TMT facility AO system NFIRAOS, and have hence generated a TT error budget. From the simulations presented in Section 4, we conclude that IR detectors are preferable to optical, which is mainly due to the expected partial correction in the IR. We find that a 2 arc min diameter patrol field for finding NGS is sufficient.

At least one NGS WFS is required to measure focus from the NGS in order to track variations in the sodium layer altitude, and we find that an additional 2 TT NGS WFS significantly improves the TT error for NFIRAOS. The best TT estimate however is gained by using a Rayleigh LGS in conjunction with a TT NGS and the sodium LGS asterism. We have discarded this option for now, due to the optical complexity of using LGS at two different altitudes.

The current TMT design is for the NGS WFS sensors to be included in the science instruments. Therefore the chosen number and design of TT sensors may vary for the different science instruments, depending on how much TT jitter is acceptable in each case.

Simulation results also indicate a significant improvement in using a matched filter approach to estimating TT from the NGS, rather than with a quad-cell Shack-Hartmann. IR detector development will be necessary to implement this option.

\section{Acknowledgements}

The authors gratefully acknowledge the support of the TMT partner institutions. They are the Association of Canadian Universities for Research in Astronomy (ACURA), the Association of Universities for Research in Astronomy (AURA), the California Institute of Technology and the University of California. This work was supported, as well, by the Canada Foundation for Innovation, the Gordon and Betty Moore Foundation, the National Optical Astronomy Observatory, which is operated by AURA under cooperative agreement with the National Science Foundation, the Ontario Ministry of Research and Innovation, and the National Research Council of Canada.

\section{REFERENCES}

1. L. M. Stepp, S. E. Strom, "The Thirty-Meter Telescope project design and development phase,"in Second Backaskog Workshop on Extremely Large Telescopes, A. L. Ardeberg, T. Andersen, Eds., Proc. SPIE 5382, ,67-75 (2004).

2. E. T. Brunetto, P. Dierickx, R. Gilmozzi, M. Le Louarn, F. Koch, L. Noethe, C. Verinaud, N. Yaitskova, "Progress of ESO's 100-m OWL optical telescope design," in Second Backaskog Workshop on Extremely Large Telescopes, A. L. Ardeberg, T. Andersen, Eds., Proc. SPIE 5382, 159-168 (2004). 
3. M. W. Johns, "Giant Magellan Telescope (GMT)," in Second Backaskog Workshop on Extremely Large Telescopes, A. L. Ardeberg, T. Andersen, Eds., Proc. SPIE 5382, 85-94 (2004).

4. T. E. Andersen, A. Ardeberg, H. Riewaldt, M. Lastiwka, N. Quinlan, K. McNamara, X. Wang, A. Enmark, M. Owner-Petersen, A. Shearer, C. Fan, D. Moraru, "Status of the Euro50 Project," in Ground-based Telescopes, J. M. Oschmann Ed., Proc. SPIE 5489, 407-416 (2004).

5. H. W. Babcock, "The Possibility of compensating astronomical seeing," Pub. Astron. Soc. Pac. 65, 229-236 (1953).

6. D. B. Calia, B. L. Ellerbroek, and R. Ragazzoni, eds., Advancements in Adaptive Optics, Proc. SPIE $\mathbf{5 4 9 0}$ (2004).

7. B. L. Ellerbroek and F. R. Rigaut, "Methods for correcting tilt anisoplanatism in laser-guide-star-based multiconjugate adaptive optics," J. Opt. Soc. Am. A 18, 2539-2547 (2001).

8. M. Le Louarn and M. Tallon, "Analysis of modes and behavior of a multiconjugate adaptive optics system," J. Opt. Soc. Am. A 19 912-925, (2002).

9. B. Femenia, "Tip-tilt reconstruction with a single dim natural guide star in multiconjugate adaptive optics with laser guide stars," J. Opt. Soc. Am. A 22, 2719-2729 (2005).

10. D. J. Butler, R. I Davies, H. Fews, R. M. Redfern, N. Ageorges, W.K. Hackenberg, R-R. Rohloff, S. Rabien, T. Ott, S. Hippler, "Sodium layer monitoring at Calar Alto by LIDAR," in Adaptive Optical Systems Technology, P. L. Wizinowich Ed., Proc. SPIE 4007, 358-367 (2000).

11. R. M. Clare and B. L. Ellerbroek, "Sky Coverage estimates for adaptive optics systems from computations in Zernike space," J. Opt. Soc. Am. A 23, 418-426 (2006).

12. R. M. Clare and B. L. Ellerborek, "Adaptive optics sky coverage modeling for the Thirty Metre Telescope," in preparation.

13. G. Herriot, P. Hickson, B. L. Ellerbroek, D. A. Anderson, T. Davidge, D. A. Erickson, I. P. Powell, R. Clare, M. Smith, L. Saddlemyer, J.-P. Véran, "NFIRAOS: TMT facility adaptive optics with conventional DMs," in Astronomical Adaptive Optics Systems and Applications II, R. K. Tyson, M. Lloyd-Hart; Eds., Proc. SPIE 5903 1-11 (2005).

14. R. Noll, "Zernike Polynomials and atmospheric turbulence," J. Opt. Soc. Am. A 66, 207-211 (1976).

15. J. N. Bahcall and R. M. Soneira, "The universe at faint magnitudes. I. Models for the galaxy and the predicted star counts," Astrophys. J. Supplement 44, 73-110 (1980).

16. A. Spagna, "Guide star requirements for NGST: deep NIR starcounts and guide star catalogs," STScINGST-R-OO13B (2001).

17. B. L. Ellerbroek, "Wavefront reconstruction algorithms and simulation results for multi-conjugate adaptive optics on giant telescopes," Proc. SPIE 5382, 478-489 (2003).

18. J-P. Véran, G. Herriot, "Woofer-Tweeter tip-tilt control for NFIRAOS for TMT," to appear in Advances in Adaptive Optics II, B. L. Ellerbroek, D. B. Calia; Ed.s, Proc. SPIE 6272 (2006).

19. G. Angeli, Systems Engineer, TMT project, Pasadena Ca (Personal Communication, 2005).

20. G. Herriot, P. Hickson, B. L. Ellerbroek, C. Y. She, "Focus errors from tracking sodium layer altitude variations with laser guide star adaptive optics for the Thirty Metre Telescope," to appear in Advances in Adaptive Optics II, B. L. Ellerbroek, D. B. Calia; Ed.s, Proc. SPIE 6272 (2006).

21. J. Vernin, A. Agabi, R. Avila, M. Azouit, R. Conan, F. Martin, E. Masciadri, L. Sanchez, and A. Ziad, "1998 Gemini site testing campaign: Cerro Pachon and Cerro Tololo," Gemini Document RTP-AO-G0094 (Gemini Observatory, Hilo, Hawaii, 2000).

22. D. L. Fried, "Optical Resolution through a randomly inhomogenous medium for very long and very short exposures," J. Opt. Soc. Am. A 56, 1376-1379 (1966).

23. "Observing Constraints - Sky Background,"

http://www.gemini.edu/sciops/ObsProcess/obsConstraints/ocSkyBackground.html 